ACTA SCIENTIFIC DENTAL SCIENCES (ISSN: 2581-4893)

Volume 3 Issue 10 October 2019

Short Communication

\title{
Dental Amalgam Safety
}

\section{Juan Giuseppe Castillo Márquez*}

Department of Odontology, Peru

*Corresponding Author: Juan Giuseppe Castillo Márquez, Department of Odontology, Peru.

Received: August 30, 2019; Published: September 25, 2019

DOI: 10.31080/ASDS.2019.03.0650

Dental amalgam (silver fillings) has been used for over than 150 years in order to effectively restore decay teeth, and it's still used due to its excellent strength and durability. Indeed millions of individuals are using dental amalgams nowadays, nevertheless there is also concerns about its biological safety specifically by the fact that dental amalgam contains a liquid metal called mercury. Even though virtually all people have at least minute amounts of methylmercury in their body dental amalgam it's saying could be favoring the mercury body accumulation and for this reason it is suspicious for threat health in some subpopulations.

First of all, dental amalgam is an alloy of metals which contain a liquid metal called mercury. In general terms any alloy which contain mercury it's called "amalgam". Dental amalgam is compound by $\sim 50 \%$ of mercury and $\sim 50 \%$ by other metals such as silver, copper and tin. It has been used for restored tooth decay for more than 150 years, and it becomes very popular due to its strength to support all different mastication forces among life years. Dental amalgam it's an alloy that carry out all requirements to restore a dental cavity due to caries. When a dentist blend mercury, silver and copper this results in a very hard union which at first become smooth and fill the cavity but then it becomes very hard so can last for pretty years or even though whole life. Nevertheless mercury contains in it is not stable. Mercury contain in dental amalgams is gradually liberated to the body each time we stimulate its separation. What is really a concern respect of this alloy is whether it liberates safety mercury amounts to our body through the years.

Secondly, it's said that certain subpopulations could be in more danger than another one. None of us have the same metabolic reaction to one substance, hence something that couldn't be dangerous for someone could do for others one; such as is said in a study leading by Homme., et al it's possible that some subpopulations are in higher risk than another ones in front to the same substance, in this case from mercury contain in dental amalgams, based on their epigenetic variability. Not everyone whose are using dental amalgam are in risk. Even though it's known that "dental amalgam releases low levels of elemental mercury vapor" there is still discussion regarding if these levels of vapor are safe as the most important North American authorities such as the Food and Drug Administration (FDA) and the American Dental Association (ADA) support dental amalgam as a safe and effective material for dental restorations" [1]. What really is in discussion is if there is health's hazard risk all over the world by using dental amalgam in restoring tooth decay despite of its strength and durability.

A third consideration was if mercury in dental amalgam could be spreading by using other technologies existing nowadays. Electro Magnetic Fields (EMF) was not favorable for persons whom use dental amalgams. Like a study lead by Mortazavi., et al. says EMF can be found in some devices very used nowadays like mobile phones, laptops and medical equipments like Magnetic Resonance Image (MRI), yet these devices are able to liberate mercury from dental amalgams and this liberation could be higher if amalgam was putted 24 hours before an exposure to MRI. So Electro Magnetic Fields could lead to release mercury from this dental alloy. This devices which function by EMF "release mercury from dental amalgam fillings in individuals who used mobile phone" (Mortazavi., et al) [2] and this fact would aid to reinforce the idea that mercury is a health hazard when is needed to used devises which function with EMF. Nevertheless this studies are not determinants and "no unanimous agreements exists among investigators on the amount of released mercury from den $\neg$ tal amalgam that under normal circum $\urcorner$ stances, would cause a toxic response in humans" [2].

Citation: Juan Giuseppe Castillo Márquez. “Dental Amalgam Safety”. Acta Scientific Dental Sciences 3.10 (2019): 73-74. 
Moreover, there is an environmental concern about dental amalgams. Amalgam environmental pollution is not really a problem. Even though there is a real worried about contamination for human health from dental amalgam use it represents a very minor contribution to total human exposure from soil and through Inhalation" [3]. Nevertheless the Scientific Committee on Health and Environmental Risks (SCHER) also say that at present, there is no scientific evidence supporting and endorsing these statements. Therefore, more research on alternative materials [for dental amalgam] is recommended.

Finally, dental amalgam will be scrutinized in order to know about its safety. Every time more organizations give their opinion about its safety. For instance the World Health Organization (WHO) says that a world short-term prohibition of dental amalgam will set public health and dentistry problems, but indeed is convenient to follow a gradual elimination favoring prevention and alternatives for dental amalgam. But also establishes a range or threshold. Thus the WHO "estimates that the typical absorbed dose of mercury from amalgams is $1-22$ micrograms per day, with most people incurring doses of less than 5 micrograms per day" [1].

To sum up, dental amalgam has to be scrutinized in order to get a sound knowledge about its safety since not all people have the same metabolic reaction to the same substance, furthermore it's now known that there are subpopulations which would be in higher risk than another one and mayor studies will determine whether EMFs technologies would affect people which have recent dental amalgam restorations but regarding of environmental pollution from using dental amalgam it doesn't represent really the essence of the problem.

\section{Bibliography}

1. Kristin G Homme., et al. "New science challenges old notion that mercury dental amalgam is safe". Biometals 27.1 (2014): 19-24.

2. Mortazavi SMJ., et al. "High-field MRI and mercury release from dental amalgam fillings". International Journal of Occupational and Environmental Medicine 5.2 (2014): 101-105.

3. Scientific Committee SCHER., et al. "Opinion on environmental risks and indirect health effects of mercury from dental amalgam". ResearchGate. Elsevier (2015).

\section{Volume 3 Issue 10 October 2019 \\ (C) All rights are reserved by Juan Giuseppe Castillo Márquez.}

